Polymer Journal, Vol. 39, No. 8, pp. 834-840 (2007)

(C) 2007 The Society of Polymer Science, Japan

\title{
Macroscopic Deformation and Failure of Ductile Polyethylene: The Dominant Role of Entangled Amorphous Network
}

\author{
Bing NA, ${ }^{1, \dagger}$ Ruihua Lv, ${ }^{1}$ Qin ZHANG, ${ }^{2}$ and Qiang $\mathrm{FU}^{2}$ \\ ${ }^{1}$ Department of Materials Science and Engineering, East China Institute of Technology, \\ Fuzhou, 344000, People's Republic of China \\ ${ }^{2}$ Department of Polymer Science \& Materials, Sichuan University, State Key Laboratory of Polymer Materials Engineering, \\ Chengdu, 610065, People's Republic of China
}

(Received November 28, 2006; Accepted May 15, 2007; Published June 26, 2007)

\begin{abstract}
Ductile polyethylenes with various molecular weights have been adopted to stress the dominant role of entangled amorphous network in the macroscopic deformation and failure behaviors. The true draw ratio at break and permanent strain of broken samples after total shrinkage at $140{ }^{\circ} \mathrm{C}$ for $10 \mathrm{~min}$ demonstrate that entangled amorphous network undergoes more significant relaxation in the sample with lower molecular weight. With aid of Essential Work of Fracture (EWF) method, most of importance, the fracture toughness of ductile polyethylene is effectively evaluated and can be directly related to the network modulus. Furthermore, fracture toughness is strain rate dependent and can be enhanced to some extent by suppressing relaxation of entangled network. [doi:10.1295/polymj.PJ2006175] KEY WORDS Entangled Network / Deformation / Fracture Toughness / Polyethylene /
\end{abstract}

As a partially crystalline polymer, polyethylene has a two-phased structure with crystalline and amorphous domains arranged alternatively. With extensive efforts, the role of crystalline component in the deformation of polyethylene has been disclosed to large extent. ${ }^{1-8}$ Lamellar slip, adopted from the theory of crystal plasticity, dominates the yield process of polyethylene. With increasing strain, subsequently, lamellae are gradually destroyed and thus finally a fibrillar structure is brought up. In the past, however, the effect of amorphous component on the deformation of polyethylene is underestimated due to its fluid property at or above room temperature. Actually, the post yield deformation at large strains is mostly taken over by the amorphous component rather than the crystalline counterpart. ${ }^{9-13}$ Many experiments have demonstrated that the deformation of the partially crystalline polyethylene may be regarded as the stretching of an entangled network that is represented by trapped chain entanglements in the amorphous phase and crystallites acting as physical cross-links. The deformation of an entangled network can be further accommodated in the framework of rubber elasticity, which has been well highlighted in the works of Harwad. ${ }^{14}$ The entangled network could manifest itself in high reversibility of the deformation when the drawn samples are heated up to its melting point. However, the results from Strobl's group indicate that a loss of memory of entangled network can occur while the topology of entangled network changes. ${ }^{9}$ In other words, a partial relaxation of the entangled network will lead to permanently viscous flow and thus total recovery of strain becomes impossible. This can be easily understood since physical cross-links from the entanglements, tie-molecules and crystallites adjacent to the amorphous layers in polyethylene with a high viscosity can be resolved so long as the deformation rate is comparable to its relaxation time.

Besides the yield and post yield phenomena, the failure and toughness are another challenging topics in research, since they establish a basis for the end use of the polymer. Long ago, brittle to ductile transition induced by a molar mass in tensile stretching is recognized. ${ }^{15-17}$ Firstly, the effect of crystallinity and supermolecular structure is emphasized to give a plausible explanation for the origin of brittle to ductile transition. Later, the importance of tie molecule and entanglement is gradually disclosed. High molecular weight ensures high network modulus and thus premature failure of a sample could be precluded. Similar to the explanations used in tensile tests, the chain entanglement density is also the major parameter in understanding the impact toughness of polyethylene. ${ }^{18,19}$ Though the failure of polyethylene is related to the chain entanglements to some extent for explaining the brittle ductile transition, the relationship between them is still qualitative, especially when samples are solely deformed in the ductile region. Moreover, the effect of relaxation of entangled network on the fracture toughness has not been explored yet. In this research, the dominant role of entangled network in the macroscopic deformation and failure of ductile polyethylene will be investigated with aid of tensile tests and Essential Work of Fracture (EWF) tests.

${ }^{\dagger}$ To whom correspondence should be addressed (Fax: 0086-794-8258320, E-mail: bna@ecit.edu.cn, bingnash@163.com). 
Table I. The characteristic parameters of ductile polyethylene used in this study. The melting characteristics are derived from differential scanning calorimetry (DSC) heating runs at a rate of $10^{\circ} \mathrm{C} / \mathrm{min}$. For the calculation of crystallinity a value of $293 \mathrm{~J} / \mathrm{g}$ for the heat of fusion at $100 \%$ crystallinity is used

\begin{tabular}{ccccccc}
\hline Materials & $\begin{array}{c}\text { Sample } \\
\text { code }\end{array}$ & $M_{\mathrm{w}}(\mathrm{g} / \mathrm{mol})$ & $M_{\mathrm{w}} / M_{\mathrm{n}}$ & $\begin{array}{c}\text { Melting point } \\
\left({ }^{\circ} \mathrm{C}\right)\end{array}$ & Crystallinity & $\begin{array}{c}G \\
(\mathrm{MPa})\end{array}$ \\
\hline \multirow{3}{*}{ HDPE } & $\mathrm{H} 1$ & $7.3 \times 10^{4}$ & 5.9 & 132.6 & 61.6 & 1.2 \\
& $\mathrm{H} 2$ & $1.4 \times 10^{5}$ & 8.6 & 132.2 & 60.3 & 1.5 \\
UHMWPE & $\mathrm{H} 3$ & $3.3 \times 10^{5}$ & 8.4 & 131.5 & 55.7 & 2.0 \\
\hline
\end{tabular}

Furthermore, the relationship between entangled network and fracture toughness will be explored to some extent.

\section{EXPERIMENTAL SECTION}

\section{Materials and sample preparation}

Three high-density polyethylenes (HDPE) and one ultra-high molecular weight polyethylene (UHMWPE) were adopted in the study. Their characteristics were present in Table I. Samples for the mechanical tests were prepared in a hot press with a temperature of $200^{\circ} \mathrm{C}$ and a pressure of $2 \mathrm{MPa}$. After held for $5 \mathrm{~min}$ in the press, samples were quickly transferred to a cold press for rapid cooling under a slight pressure. Film thicknesses were about $0.5 \mathrm{~mm}$.

\section{Mechanical tests}

Tensile tests were performed on the dog-bone samples $(4 \mathrm{~mm}$ width $\times 6 \mathrm{~mm}$ gauge length) using an universal testing machine (WDT II) equipped with a $200 \mathrm{~N}$ load cell at room temperature. Due to necking effect, true draw ratio $\lambda$ was obtained from the separation of ink marks at regular $0.2 \mathrm{~mm}$ intervals preprinted on the specimens with aid of a CCD camera (1280 pixel $\times 1024$ pixel) equipped with a tunable magnification lens. The image resolution was 80pixels $/ \mathrm{mm}$. Moreover, with assumption of a constant volume in tensile, ${ }^{9}$ true stress $\sigma$ could be determined by adopting the equation

$$
\sigma=\sigma_{n} \times \lambda
$$

where $\sigma_{n}$ denotes the nominal stress or engineering stress.

Fracture toughness tests were conducted by tensile deformation of the deeply double edge-notched strips with a gauge length of $10 \mathrm{~mm}$ and a width of $15 \mathrm{~mm}$. Initial notches were made perpendicularly to the tensile direction with a fresh razor blade. The above procedure for characterization of toughness was referred to the concept of Essential Work of Fracture (EWF) developed recently. ${ }^{20-22}$ It had two major advantages: one was that the toughness of ductile polymers could be effectively evaluated due to complete fracture of samples, unlike the situation in common Izod impact tests; the other was that the energy associated with the separation of a crack, which was the intrinsic parameters of samples, could be isolated from the total energy consumed during the deformation. In brief, a plot of specific work of fracture $w_{f}$, consumed by per unit area of ligament, as a function of ligament length $l$ should be a linear relation. Its interception at zero ligament length and its slope would give the specific essential work of fracture $w_{e}$ and the specific non-essential work of fracture $\beta w_{p}$, respectively. For meeting the demands of plane stress and free constraint of boundary, the ligament lengths of specimens were varied between 2.5 and $5 \mathrm{~mm}$. The ligament lengths were accurately measured during initial deformation stage with aid of the CCD camera mentioned above. With real time registration of images, it was possible to directly correlate the two dimensional deformation state to the characteristic points of the loaddisplacement curve. The load-displacement curves were recorded during deformation, and the absorbed energy was calculated by computer integration of the loading curves.

\section{Free shrinkage tests}

To study the memory of deformed entangled network, broken samples were first placed in an oven with a temperature of $140^{\circ} \mathrm{C}$ for $10 \mathrm{~min}$. Thereafter, the final extension ratio of ink marks, preprinted on the samples before deformation, was registered by the above CCD camera and denoted as the permanent strain.

\section{RESULTS AND DISCUSSION}

\section{Network properties}

To learn about the network properties, a simple route devised by Haward and Thackray is followed. ${ }^{14}$ They suggested that the deformation of a partially crystalline polymer could be modeled by a simple constitutive model shown in the Figure 1. It consists of a Hookean spring, the initial tensile modulus $E$, connected in series with a dashpot being in parallel with another non-linear spring that represents the 


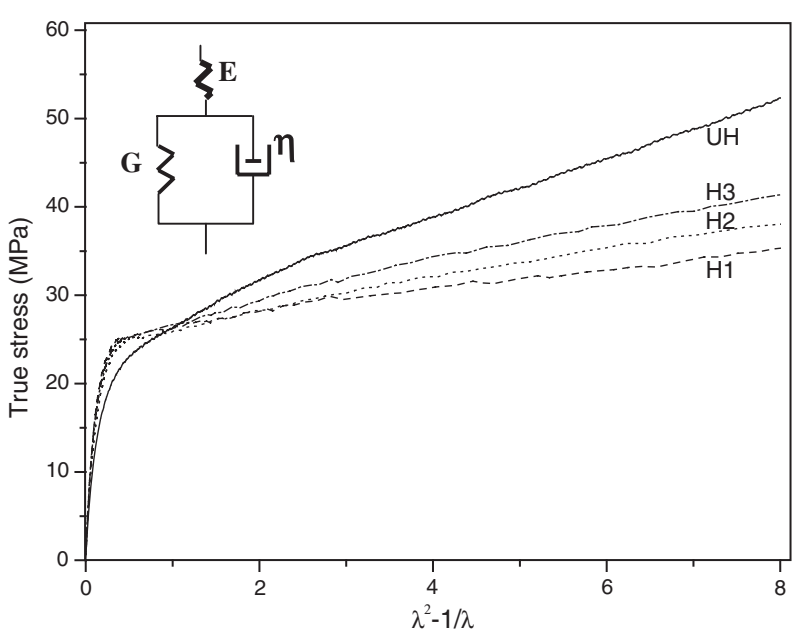

Figure 1. Haward-Thackray plot of linear polyethylene at a nominal strain rate of $0.0025 \mathrm{~s}^{-1}$. The model is shown as an inset.

shear modulus of the network $G$. Plots of true stress $\sigma$ versus $\lambda^{2}-1 / \lambda$ yield two slopes, one at the initial, giving $E$, and the other at larger strains, giving $G$. Moreover, from the results presented by Strobl et al., ${ }^{9}$ we know that, so long as the draw ratio is no more than about 3 , little molecular relaxation within network occurs and thus a complete reversibility of deformation can be brought up when deformed partially crystalline polyethylene is brought up to its melting point. Therefore, the network properties of polyethylene can be successfully extracted from a HawardThackray plots shown in Figure 1. The obtained shear modulus of network are ranged from 1.2 to $3.3 \mathrm{MPa}$ and correspond with those found by Harward, Strobl and Bartczak et al. ${ }^{9,14,24}$

\section{Failure and toughness}

Figure 2 is the nominal stress strain curves of four linear polyethylenes at a nominal strain rate of $0.0025 \mathrm{~s}^{-1}$. As expected, all samples exhibit ductile behaviors in tensile. Moreover, upturn of stress at large strain before fracture becomes more obvious with increasing molecular weight. This is in that the less relaxation of the amorphous network with increasing molecular weight can build up the stress more easily and thus earlier fracture of sample is brought up. Interesting to note that, as for sample H1 with the lowest molecular weight, the stress cannot build up during the whole deformation. The true draw ratio at break, in situ registered by CCD camera, is shown in Figure 3 for all four samples. The highest extension, up to about 30 , is found in the sample $\mathrm{H} 1$ and draw ratio is decreased significantly with increasing molecular weight. In addition to the stretching and extension of a molecular network, here we also pay attention to the relaxation of stretched network during deformation. The permanent strain

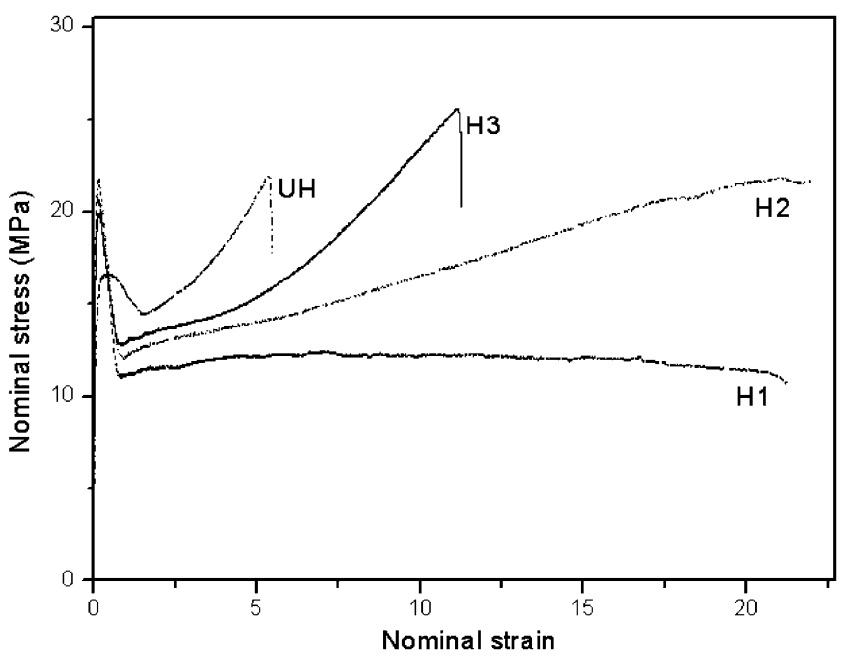

Figure 2. Nominal stress strain curves of linear polyethylene at a nominal strain rate of $0.0025 \mathrm{~s}^{-1}$.

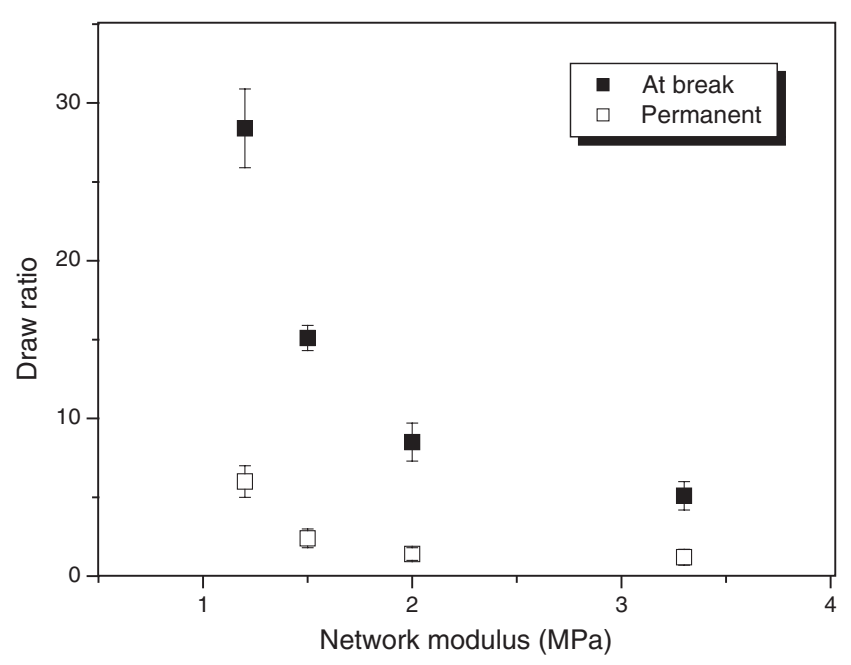

Figure 3. Draw ratios, determined by the separation of ink mark preprinted on the samples with CCD camera, of linear polyethylene subjected to a nominal strain rate of $0.0025 \mathrm{~s}^{-1}$. The values at break and after free shrinkage at $140{ }^{\circ} \mathrm{C}$ for $10 \mathrm{~min}$ are included.

of broken samples after free shrinkage at $140{ }^{\circ} \mathrm{C}$ for $10 \mathrm{~min}$ is also present in Figure 3. Similar to the draw ratio at break, the permanent strain decreases sharply with increasing molecular weight. Moreover, for the sample $\mathrm{H} 3$ and $\mathrm{UH}$, almost total recovery is achieved, which indicates that nearly no relaxation of molecular network occurs during tensile deformation. The reason lies in that it will take more time to pull out of a chain from the entangled network with higher molecular weight. ${ }^{12}$ The above results demonstrate that, though the high extension is not easily achieved in sample with higher molecular weight, the relaxation of molecular network during stretching can also be suppressed to large extent.

Figure 4 is two dimensional deformation states of 

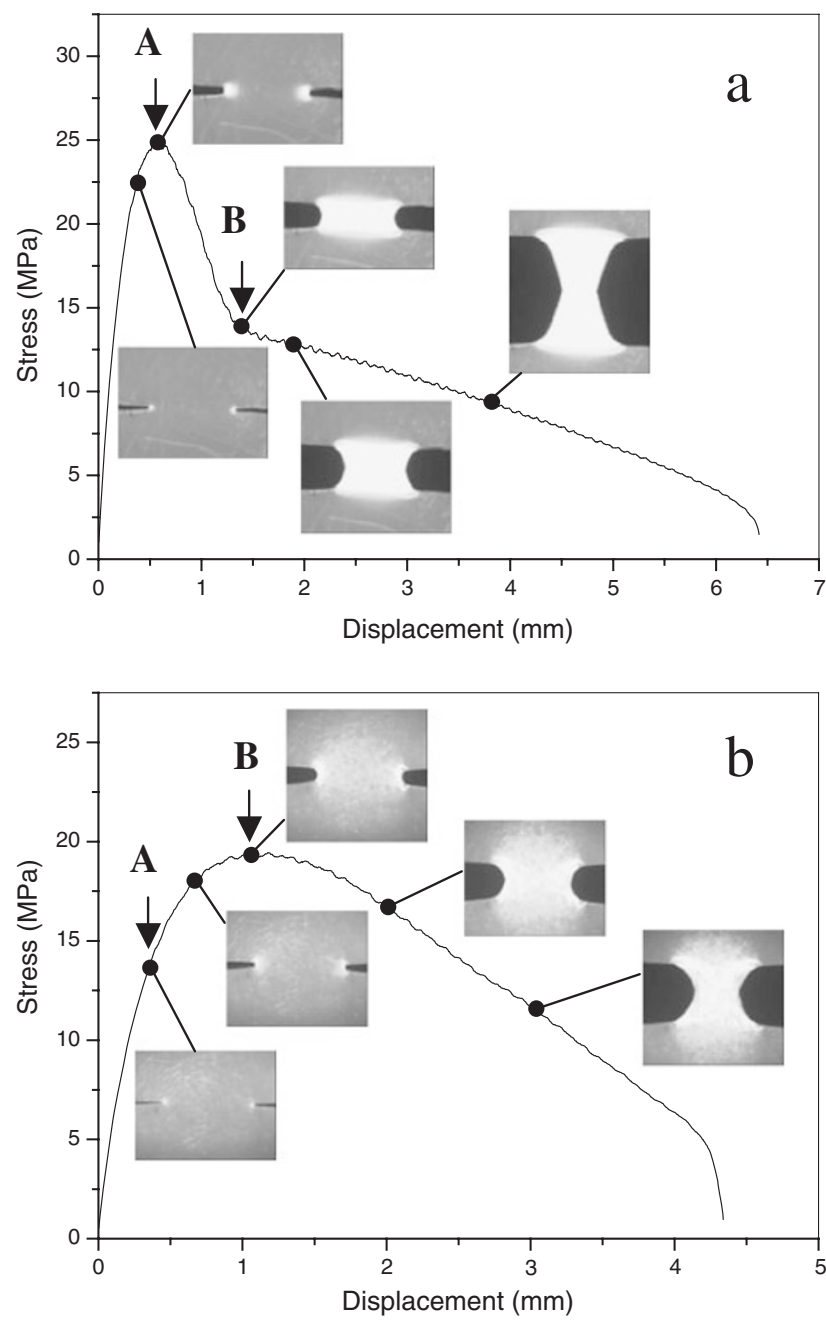

Figure 4. Correlation of two-dimensional states to the stress displacement curves of sample $\mathrm{H} 2$ (a) and $\mathrm{H} 4$ (b), respectively, with deeply double edge notches at a nominal strain rate of $0.0025 \mathrm{~s}^{-1}$.

deeply double edge-notched samples $\mathrm{H} 2$ and $\mathrm{UH}$ with a ligament length of $3.6 \mathrm{~mm}$, respectively, subjected to tensile deformation at a nominal strain rate of $0.0025 \mathrm{~s}^{-1}$. Note that the point A and point B in the Figure 4 denote the onset of yield and crack propagation, respectively (see below). Apparently, in these two cases, yield is first initiated from the notches due to stress concentration and stress-whitening zones are formed ahead of notches. With increasing of elongation, stress-whitening zones begin to grow from both sides and then merge into each other. After total yield of whole ligament, crack propagation sets in until the ultimate fracture of samples. It should be mentioned that the stress-displacements of all HDPE samples, including $\mathrm{H} 1, \mathrm{H} 2$ and $\mathrm{H} 3$, are similar. With real time registration of ligament width, moreover, the relation of crack growth versus time can be determined. Figure 5 is, for example, such a plot derived from sample $\mathrm{H} 2$ with a ligament length of $3.6 \mathrm{~mm}$.
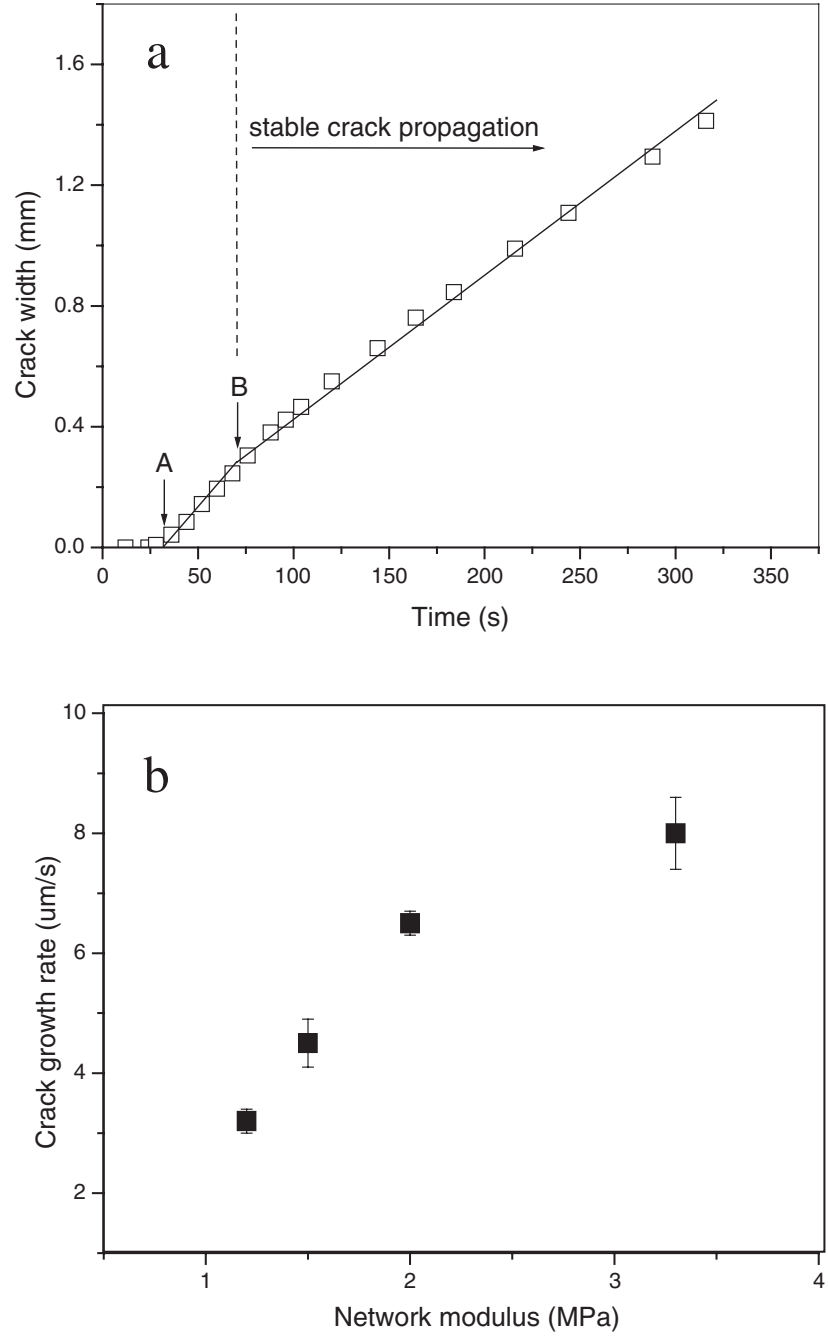

Figure 5. Plots of the crack width versus time of sample H2 with deeply double edge notches at a nominal strain rate of $0.0025 \mathrm{~s}^{-1}$ (a) and the relation of crack growth rate to the network modulus for all linear polyethylene (b).

Consistent with the results of two-dimensional deformation state, there have three distinct zones during the whole tensile deformation. In the first zone no crack grows, followed by the sharp increase of crack width due to yield of the whole ligament in the second zone, and finally in the third zone a stable crack propagation sets in. From the arguments presented in the introduction section, we are aware that the deformation is mostly dominated by the entangled network at large strain, therefore, a plot of crack growth rate versus network modulus, shown in Figure 6, could give some instructive results. The crack growth rate increases with network modulus, which means that rapid crack growth is brought up in the less relaxed sample, i.e. UHMWPE (UH). In other words, significant crack blunting effect occurs in the more relaxed sample with lower molecular weight. Since the glass transition temperature of linear polyethylene is well below the room temperature, the mobility of chains in the entan- 


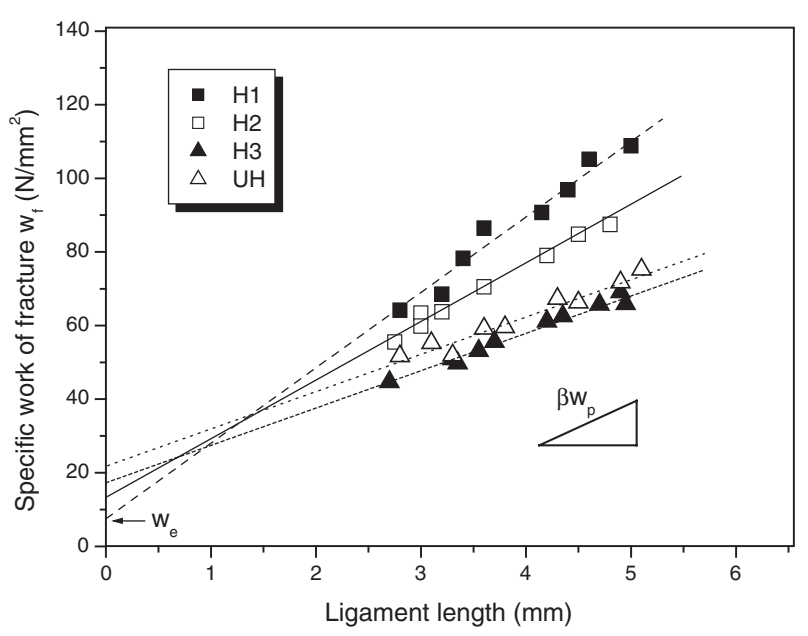

Figure 6. Plots of specific work of fracture $w_{f}$ as a function of ligament length, obtained from all four samples with deeply double edge notches at a nominal strain rate of $0.0025 \mathrm{~s}^{-1}$. Its interception at zero ligament length and its slope would give the specific essential work of fracture $w_{e}$, and the specific non-essential work of fracture $\beta w_{p}$, respectively.

gled amorphous region is high enough to be easily deformed under tensile stretching. Moreover, with suitable entanglements the molecular network can sustain the external force and the brittle facture can be effectively prevented, which is the situation even for the sample H1 with the lowest molecular weight. During crack propagation, therefore, the chains disengaged from each other across the fracture plane must be firstly stretched within the entangled network, and the cohesive strength is determined by the covalent bonds rather than by van der Walls forces among molecular chains. While the cohesive strength exceeds the network modulus, a crack will blunt instead of propagating, ${ }^{25}$ consistent with our results. Only while chains within entangled network become highly stretched can a crack grow. Based on the results shown in Figure 2, it is evident that the chains in the less relaxed sample, for example, UH, can be extended rapidly and thus promotes the crack growth.

Since the stretching and relaxation of entangled network have significant effect on the crack propagation, it is natural to explore the relationship between energy consumed to form the fracture plane and entangled network properties. As suggested by the EWF method, a plot of total energy dissipated on per unit area of ligament $w_{f}$ versus ligament length could yield specific essential work of fracture $w_{e}$, an intrinsic parameter associated with fracture toughness, and the specific non-essential work of fracture $w_{p}$, energy dissipated by the plastic flow in the outer zone, respectively. ${ }^{20-23}$ Such plots for four polyethylenes are present in the Figure 6. As for the same ligament, the total energy dissipated on per unit area of ligament $w_{f}$ increases

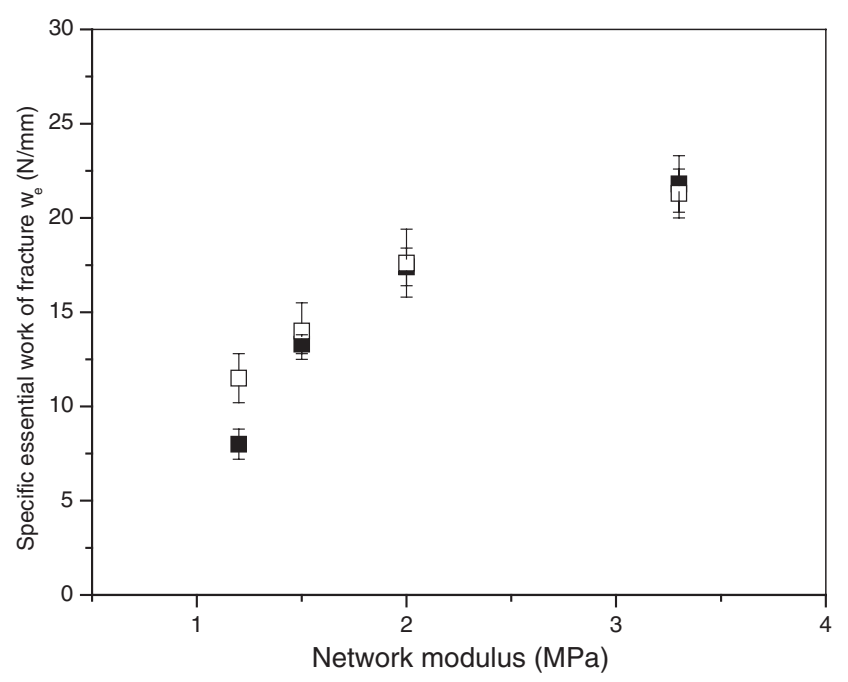

Figure 7. Plots of specific essential work of fracture $w_{e}$, obtained at a strain rate of $0.0025 \mathrm{~s}^{-1}$ (solid symbol) and $0.125 \mathrm{~s}^{-1}$ (open symbol), respectively, versus network modulus for all four polyethylenes.

with decreasing molecular weight. From the interception of fitted lines at zero ligament length, however, an opposite tendency can be obtained. Moreover, a close inspection shows that the specific essential work of fracture $w_{e}$ increases with network modulus, as shown in the Figure 7. It means that the fracture toughness can be correlated with entangled amorphous network. It is desired to note that the fracture toughness mostly depends on the deformation resistance of oriented fibrils ahead of cracks when the random lamellae are gradually converted into highly oriented fibrils at large strain. ${ }^{26}$ According to the results shown in Figures 2 and 4, it is apparent that deformation resistance of oriented fibrils is mostly determined by entangled amorphous network rather than by crystalline phase. Since the network modulus mostly depends on the relaxation of deformed chains, furthermore, it can be deduced that $w_{e}$, the energy consumed by separation of a crack, is related to the density of stretched chains across fracture plane. Note that application of EWF concept to such relaxed chains has been confirmed in evaluating the temperature-dependent toughness of isotactic polypropylene and its copolymers. ${ }^{27}$ Due to significant relaxation of entangled network is present in the sample H1, therefore, it could be expected that the density of stretched molecular chains that can afford the load is reduced significantly during fracture and thus the lowest fracture toughness is derived. Rather, severe stretching and relaxation of entangled network will lead to increase of energy dissipated in the zone out of the fracture plane. As shown in Figure 6, the specific non-essential work of fracture $\beta w_{p}$, determined from the slop of fitted lines, increase with decreasing of network modulus. 

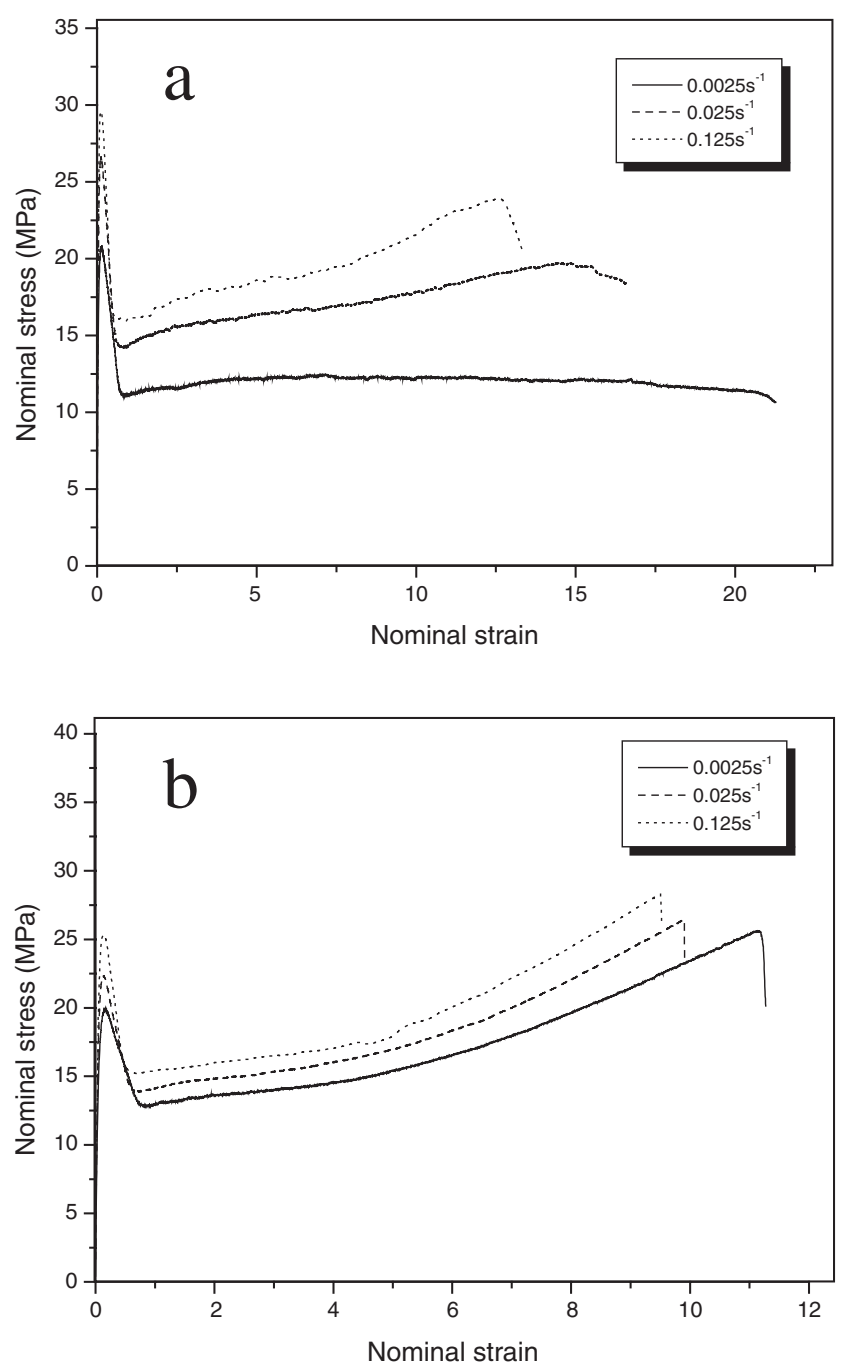

Figure 8. Nominal stress strain curves of sample H1 (a) and $\mathrm{H} 3$ (b), respectively, at the indicated nominal strain rate.

\section{Effect of strain rate}

Since relaxation of molecular network is time-dependent, naturally, various strain rates could throw some effects on the deformation and failure of polyethylene. Figure 8 is the nominal stress strain curves of sample $\mathrm{H} 1$ and $\mathrm{H} 3$, respectively, at various nominal strain rates. As expected, stress upturn before fracture becomes apparent with increasing of strain rate in the sample $\mathrm{H} 1$, indicating that the relaxation of entangled network is suppressed to some extent. As for sample $\mathrm{H} 3$ with higher network modulus, however, the effect of strain rate on the accumulation of stress is indiscernible. The dependence of relaxation of entangled network on the strain rate can be further illustrated by the permanent strain of broken samples, as shown in Figure 9. Consistent with the results of stress strain curves, permanent strain is more sensitive to strain rate in the sample with lower molecular weight. The effect of strain rate on the fracture toughness is also explored. As presented in Figure 10, evidently, the de-

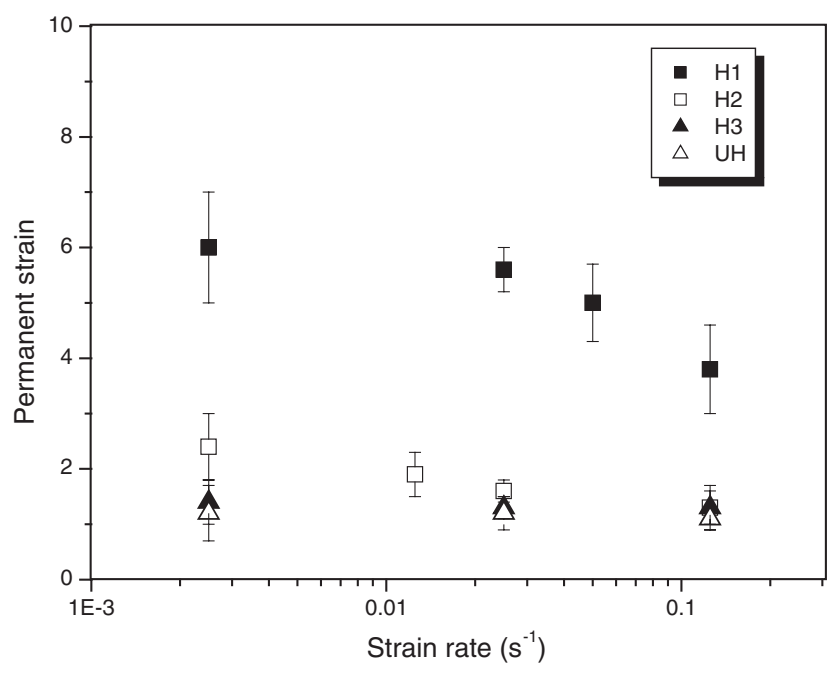

Figure 9. Permanent strain after free shrinkage at $140{ }^{\circ} \mathrm{C}$ for 10 min, determined by the separation of ink mark preprinted on the samples with CCD camera, of linear polyethylene subjected to various nominal strain rates.

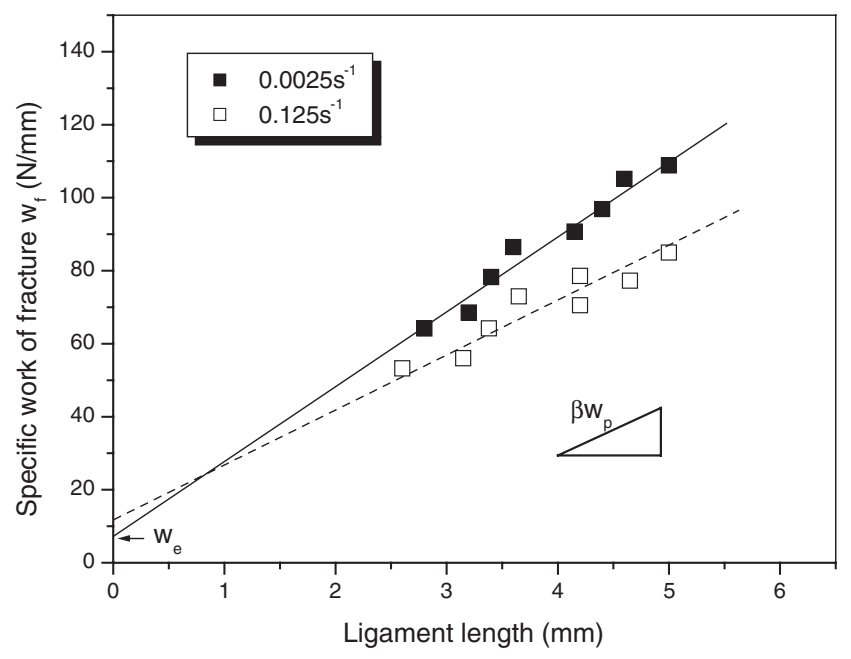

Figure 10. Plots of specific work of fracture $w_{f}$ as a function of ligament length, obtained from sample $\mathrm{H} 1$ with deeply double edge notches at a nominal strain rate of $0.125 \mathrm{~s}^{-1}$. For comparison, the one obtained at a nominal strain rate of $0.0025 \mathrm{~s}^{-1}$ is also included.

pression of relaxation of entangled network, on one hand, can enhance the fracture toughness, specific essential work of fracture $w_{e}$, due to increasing of density of stretched chains across the fracture plane, and reduce the energy dissipated in the outer zone due to suppression of plastic flow, on the other hand. The deduced specific essential work of fracture $w_{e}$ of four samples at a nominal strain rate of $0.125 \mathrm{~s}^{-1}$ is included in the Figure 7. It is again demonstrated that the fracture toughness of sample with higher molecular weight is less sensitive to the strain rate due to little change of relaxation of entangled network with strain rates. 


\section{CONCLUSION}

Based on the network modulus derived from Haward-Thackray plots, the dominant role of entangled network in the deformation and failure of polyethylene are addressed in detail in this study. The extension and relaxation of entangled network with higher molecular weight can be suppressed to large extent, indicated by lower true draw ratio at break and lower permanent strain of broken samples after total shrinkage at $140^{\circ} \mathrm{C}$ for $10 \mathrm{~min}$. More important, the fracture toughness of ductile polyethylene increases with network modulus and is correlated with the relaxation of stretched chains. Suppression of relaxation in the entangled amorphous network can enhance the fracture toughness, which is demonstrated by the strain rate dependent results.

Acknowledgment. The financial supports from the Natural Science Foundation of Jiangxi, China (No. 0650009*) and the Doctoral Special Foundation of ECIT are gratefully acknowledged.

\section{REFERENCES}

1. Z. Bartczak, A. S. Argon, and R. E. Cohen, Macromolecules, 25, 5036 (1992).

2. A. Peterlin, J. Mater. Sci., 6, 490 (1971).

3. A. Galeski, Z. Bartczak, A. S. Argon, and R. E. Cohen, Macromolecules, 25, 5705 (1992).

4. P. Bowden and R. Young, J. Mater. Sci., 9, 2034 (1974).

5. R. Seguela and F. Rietsch, J. Mater. Sci. Lett., 9, 46 (1990).
6. Z. Bartczak, R. E. Cohen, and A. S. Argon, Macromolecules, 25, 4692 (1992).

7. P. B. Bowden and R. Young, J. Mater. Sci., 9, 2034 (1974).

8. L. Lin and A. S. Argon, J. Mater. Sci., 29, 294 (1994).

9. R. Hiss, S. Hobeika, C. Lynn, and G. Strobl, Macromolecules, 32, 4390 (1999).

10. Y. F. Men, J. Rieger, and G. Strobl, Phys. Rev. Lett., 91, 095502 (2003).

11. B. A. G. Schrauwen, R. P. M. Janssen, L. E. Govaert, and H. E. H. Meijer, Macromolecules, 37, 6069 (2004).

12. Z. Bartczak, Polymer, 46, 10339 (2005).

13. Z. Bartczak, Macromolecules, 38, 7702 (2005).

14. R. N. Haward, Macromolecules, 26, 5860 (1993).

15. M. A. Kennedy, A. J. Peacock, and L. Mandelkern, Macromolecules, 27, 5297 (1994).

16. M. A. Kennedy, A. J. Peacock, M. D. Failla, J. C. Lucas, and L. Mandelkern, Macromolecules, 28, 1407 (1995).

17. N. Brown and I. M. Ward, J. Mater. Sci., 18, 1405 (1983).

18. B. J. Egan and O. Delatycki, J. Mater. Sci., 30, 3307 (1995).

19. B. A. G. Schrauwen, L. C. A. Breemen, A. B. Spoelstra, L. E. Govaert, G. W. M. Peters, and H. E. H. Meijer, Macromolecules, 37, 8618 (2004).

20. J. Wu and Y. W. Mai, Polym. Eng. Sci., 36, 2275 (1996).

21. R. Lach, K. Schneider, R. Weidisch, A. Janke, and K. Knoll, Eur. Polym. J., 41, 383 (2005).

22. J. Karger-Kocsis, T. Czigany, and E. J. Moskala, Polymer, 39, 3939 (1998).

23. D. Ferrer-Balas, M. Ll. Maspoch, A. B. Martinez, and O. O. Santana, Polymer, 42, 1697 (2001).

24. Z. Bartczak and M. Kozanecki, Polymer, 46, 8210 (2005).

25. C. Y. Hui, A. Jagota, S. J. Bennison, and J. D. Londono, $P$. Roy. Soc. Lond. A Mat., 459, 1489 (2003).

26. J. Cazenave, R. Seguela, B. Sixou, and Y. Germain, Polymer, 47, 3904 (2006).

27. D. Ferrer-Balas, M. L. Maspoch, A. B. Martinez, E. Ching, R. K. Y. Li, and Y. W. Mai, Polymer, 42, 2665 (2001). 